\title{
QUALIDADE SANITÁRIA DE GRÃOS DE SOJA TIPO ALIMENTO
}

\author{
ADILSON BIZZETO1 \\ RONALDO MAURICIO LEVY \\ MARTIN HOMECHIN \\ DEONISIO DESTRO \\ LILIAN AZEVEDO MIRANDA
}

\begin{abstract}
BIZZETTO, A.; LEVY, R. M.; HOMECHIN, M. et al. Qualidade sanitária de grãos de soja tipo alimento. Semina: Ci. Agr., Londrina, v.17, n.1, p.9-14, mar. 1996.
\end{abstract}

RESUMO: O objetivo foi quantificar a incidência de fungos em grãos de soja (Glycine max (L.) Merrill) provenientes de cruzamentos de soja tipo alimento com soja tipo grão. A soja tipo alimento inclui a soja com sementes grandes, com peso de cem sementes (PCS) igual ou maior que 20 gramas (soja tipo hortaliça) e a soja com sementes pequenas, com PCS menor que 10 gramas (soja para consumo na forma de brotos ou "natto"). A soja tipo grão tem sementes de tamanho médio, com PCS entre 10 e 19 gramas, e é destinada à indústria para a produção de óleo e farelo. As amostras de sementes de 116 diferentes linhagens puras foram colhidas no campo em 1993 e avaliadas pelo método de papel filtro. Foram detectados os seguintes fungos: Aspergillus sp. (40,37\%); Penicillium sp. (18,66\%); Fusarium sp. (12,44\%); Rhizopus sp. (5,71\%); Cercospora sp. (1,87\%) e Verticillium sp. (1,52\%). Em niveis inferiores a 1,5\%, foram detectados os seguintes fungos: Alternaria sp.; Colletotrichum sp.; Trichoderma sp.; Curvularia sp.; Nigrospora sp.; Phomopsis sp.; Diplodia sp. e Monilia sp. Em um programa de melhoramento genético de soja para o consumo humano, atenção especial deve ser dada a microorganismos, especialmente aos gêneros Aspergillus sp., Penicillium sp. e Fusarium sp., uma vez que possuem capacidade para produzir substâncias que podem ser prejudiciais ao homem e aos animais.

PALAVRAS-CHAVE: Fungos, Sementes, Soja, Alimento.

\section{INTRODUÇÃO}

A soja tipo alimento deve ter algumas características diferentes da soja comum (tipo grão): maior teor de proteínas; melhor qualidade (maiores teores de aminoácidos sulfurados, metionina e cistina); menor teor de óleo; sabor adocicado; cor clara das sementes (cotilédones, tegumento e hilo com cores semelhantes) de preferência creme; baixos teores de ácidos graxos insaturados linolênico e linoléico e menores teores de lipoxigenase (VELLO, 1992).

Segundo VELLO (1992), uma característica própria da soja tipo alimento é o tamanho das sementes, avaliad̋o pelo peso de cem sementes (PCS). A soja tipo grão tem sementes de tamanho médio, com PCS variável de 10 a 19 gramas / 100 sementes. Com exceção de brotos e nattô que empregam sementes de tamanho pequeno (<10 gramas / 100 sementes), a soja tipo alimento tem sementes em geral de tamanho grande (>20 gramas / 100 sementes). Geralmente, sementes de tamanho grande têm menor proporção hipocótilo/cotilédones, e, conseqüentemente, teores menores de lipoxigenases, fato que favorece o bom sabor. Além disso, sementes grandes facilitam o processamento industrial, principalmente a retirada da casca ou tegumento. No Japão, onde a maioria dos cultivares de soja têm sementes grandes (>20 gramas/
100 sementes), ela é cultivada como hortaliça, com semeadura em canteiros e transplante de duas ou três plântulas por cova em pequenas glebas ou hortas. É comumente comercializada e consumida como hortaliça, na forma de maços de plantas com vagens imaturas (edamame). Por esta razão, a soja com sementes grandes é denominada soja tipo hortaliça (vegetable soybeans).

As sementes são suscetíveis à invasão dos fungos durante a formação, a maturação, a colheita e o armazenamento, Segundo WETZEL (1987), os fungos de armazenamento podem estar presentes não apenas como contaminantes, mas também em algumas situações como micélio dormente junto aos tecidos do tegumento. Segundo LAZZARI (1995), ao contrário dos fungos de campo, os intermediários requerem uma faixa estreita de umidade relativa entre 85 e $90 \%$. Os fungos de armazenamento, a partir da faixa de $65 \%$ de UR, instalam-se e iniciam a deterioração do produto. Após a década de 60 , tem sido dada maior importância às micotoxinas em alimentos em função da morte de 100.000 perus na Inglaterra, após terem sido alimentados com ração composta por amendoim contaminado.

Os gêneros de fungos Aspergillus, Penicillium e Fusarium são os mais freqüentemente envolvidos na contaminação e na produção de micotoxinas (SHURTLEFF, 1980). Segundo ULLSTRUP (1966), esses organismos

${ }^{1}$ Depto. de Agronomia/Universidade Estadual de Londrina, Caixa Postal 6001, Londrina, PR., CEP 86051-990.( Martim Homechin). 
são capazes de se manter em desenvolvimento em substratos com teores de umidade inferiores aos exigidos pelos fungos de campo. CLEUSTROM et al (1983) destacaram a incidência de Aspergillus flavus em forragens úmidas e grãos. Fungos $A$. parasiticus e A. flavus, produzem toxinas cancerígenas e têm sido encontrado em alimentos como milho e amendoim.

Com o intuito de manter a saúde humana e a dos animais, as taxas de aflatoxinas têm sido reguladas pela restrição no transporte e na venda de produtos contaminados e sempre resultam em perda de milhões de dólares a cada ano, para os agricultores (DIENER et al, 1987).

A soja, entre as culturas ricas em óleo na semente, apresenta excepcional resistência à infecção e à contaminação por aflatoxina de $A$. flavus. DOEHLERT et al (1993) encontraram fortes evidências de que os aldeídos voláteis, produzidos pela lipoxigenase, pode contribuir para essa resistência. Porém, em várias investigações observa-se alta incidência de Aspergillus e elevada produção de toxinas. Segundo VIRGINIA et al. (1991), a incidência de aflatoxinas produzida em soja depende da capacidade do fungo em produzir toxinas e do genótipo de soja, influenciado pelas características agronômicas. IMWIDTHAYA et al. (1987) coletaram cento e cinqüenta amostras de cereais e produtos derivados em mercados de Bangkok, durante Julho de 1983 à Abril de 1985. Quarenta amostras compreendiam produtos à base de soja. Do total, $40 \%$ dos produtos fermentados de soja continham aflatoxinas AFB1 (20 p.p.b.) e AFG1 (20 à 100 p.p.b.), e, $40 \%$ dos molhos de soja apresentaram AFB1 (20 à 170 p.p.b.) além de AFG1 (40p.p.b.).

No período de 1982 a 1983, DUTTON \& WESTLAKE (1985) também avaliaram 800 amostras de mercadorias agrícolas, fenos e silagens. A aflatoxina B1 ocorreu em $27 \%$ das amostras, provavelmente produzida por $A$. flavus e $A$. parasiticus, que ocorreram em $22 \%$ dos materiais. WINCKLOW et al (1980) verificaram a contaminação de grãos de milho com aflatoxinas, em função da presença de A. flavus. Segundo LEMKE et al (1989), as toxinas produzidas por A. flavus e A. parasiticus são detectadas visualmente mediante fluorescência azul produzida por colônia cultivadas em meio ágar-coco e submetidas à luz ultravioleta de onda longa (365 $\mathrm{nm}$ ).

O caminho metabólico de biossíntese de aflatoxinas tem sido estudado; no entanto, pouco se sabe a respeito de sua regulação. Segundo LAZZARI (1995), a melhor maneira para controlar o desenvolvimento desses fungos é a atuação sobre as condições que favorecem seu desenvolvimento.

O gênero Fusarium e suas espécies, na natureza, ocorrem como saprófitos ou como parasitas das plantas, porém, certas espécies são capazes de produzir toxinas. MARASAS et al (1984) destacaram o interesse por esse gênero, nas últimas décadas, em função das intoxicações ocorridas em animais e humanos.
Cobaias alimentadas com arroz infestado apresentaram intoxicações, com hemorragia e câncer no útero (BOSCH \& MIROCHA, 1992). FRISUAD \& FILTENBORG (1993) classificaram os fungos do gênero Penicillium com base no perfil das micotoxinas e variações de cor apresentados pelos metabólitos secundários em cromatógrafo, em meio Czapek ágar.

Os gêneros Alternaria e Phomopsis, também produtores de toxinas, não são menos importantes que os demais toxigênicos. CLEAR et al. (1989), verificou dois tipos de descoloração em sementes, sendo uma delas ocasionada por Alternaria sp. Análises feitas junto às descolorações de Alternaria $s p$. detectaram a presença da toxina denominada de HT-2.

Além dos fungos micotoxigênicos de maior importância citados, há outros associados às sementes de soja (Glycine max (L.) Merrill). A qualidade sanitária das sementes é importante pois, através delas, é que se obterão os grãos destinados à alimentação humana ou animal. Dentre os fungos que se manifestaram, dá-se destaque à Alternaria sp. obtido por BRANCÃO et al (1988), Fusarium sp., Colletotrichum dematium var. truncata, Cercospora sp. e Phomopsis sojae detectados por HOMECHIN et al (1977) e HENNING (1992), Aspergillus spp., notificado por AMARAL et al (1986) e Penicillium sp., Nigrospora sp.; Trichoderma sp., Curvularia sp., detectados por PITTIS \& MACHADO (1987), Rhizopus sp., obtido por SINCLAIR \& SHURTLEFF (1975) e Diaporthe phaseolorum f. sp. meridionalis, promotor do cancro-da-haste notificado por YORINORI (1991). Segundo YORINORI (1990), a transmissão e a detecção nas sementes é difícil, só sendo possível a visualização em papel de filtro. $O$ nível de infecção em sementes não passa de $2 \%$, porém é a forma mais eficiente de disseminação a longas distâncias.

Segundo YORINORI \& GARCIA (1971), o fungo Cercospora sojina Hara, detectado em sementes de soja do cultivar Bragg, ocasionou deformação e morte de $61,08 \%$ das sementes. Das plântulas que emergiram, $12,11 \%$ apresentaram-se perfeitamente sadias $\mathrm{e}$ $26,8 \%$ apresentaram necroses de cotilédones. Em análises patológicas, manifestaram-se os fungos Cercospora sojina, C. kikuchii, Colletotrichum dematium var. truncata e Phomopsis sojae.

O presente trabalho teve como objetivo determinar os fungos associados aos grãos de soja (Glycine $\max ($ L.) Merrill) tipo alimento, dando ênfase aos micotoxigênicos Aspergillus spp., Penicillium spp. e Fusarium spp. .

\section{MATERIAL E MÉTODO}

Para a quantificação da incidência de microrganismos em soja tipo alimento, foram utilizadas 116 amostras de linhagens puras obtidas de 41 cruzamentos e colhidas na Fazenda Escola, pertencente à Universida- 
de Estadual de Londrina, no ano agrícola de 1993/94. Cada amostra foi de 200 sementes. As sementes foram avaliadas pelo método de papel de filtro. A metodologia de assepsia de materiais utilizados neste método constitui-se da desinfestação das placas de Petri com lisoforme comercial diluído a $10 \%$, autoclavagem da água utilizada para umedecimento do papel de filtro, durante 20 minutos a $120^{\circ} \mathrm{C}$, e esterilização do papel em estufa a $160{ }^{\circ} \mathrm{C}$, por 20 minutos. O plaqueamento das sementes foi feito em presença de chama e pinças desinfestadas. É importante ressaltar que antes da desinfestação, as placas permaneceram de 48 a 72 horas em solução de hipoclorito de sódio a 10\% ("Qboa" - comercial). As sementes foram incubadas por sete dias em câmara úmida, com alternância de luz e escuro. Os fungos incidentes foram visualizados mediante utilização de microscópio binocular e estereoscópico.

\section{RESULTADOS E DISCUSSÃO}

Os 41 cruzamentos dos quais obtiveram-se 116 linhagens estão condensados na Tabela 1 .

TABELA 1- Cruzamentos e as respectivas linhagens $F_{7}$ avaliadas no ano agrícola de 1993/1994 . UEL. Londrina, PR. Semeadura em 22/12/93

\section{Cruzamento}

PI 243. $514 \times$ FT-2

PI $91725-3 \times$ Doko

PI $91725-3 \times$ FT-2

PI 229.320 x Doko

Hogyoku $\times$ Doko

Imperial $x$ Doko

Imperial $x$ FT - 2

Worlverine $x$ Doko

PI $80.459 \times$ Doko

(TK \# $5 \times$ Unknown F 7) $\times$ Doko

(KS \# 3 x Akiyoshi F 7 - 2) x Doko

(KS \# 3 x Akiyoshii F 7 - 2) $\times$ FT - 2

(TN \# 4 x PI $230.970 \mathrm{~F} 7-1$ ) xDoko

(TN \# 4 x PI 230.970 F 7 - 4) xDoko

(KS $473 \times$ SJ2 F7) $\times$ Doko

(KS $473 \times$ SJ2 F7) $\times$ FT - 2

$(64-64 \times$ KS 473) $\times$ Doko

TMV $x$ Doko

Hampton $\times$ Doko

Pluto $\times$ Doko

Pluto $x$ FT - 2

PI $230.977 \times$ Doko

PI 230.977 Sel. $x$ Doko

PI 165.676 x Doko

Araçatuba $\times$ Doko

Tamba $\times$ Doko

Late Giant $x$ Doko

Japão 1 x Doko

Japão $2 \times$ FT - 2

Miyashipoken $\times$ Doko

Biloxi $252 \mathrm{~N} \times$ Doko

Aliança Preta $x$ Doko

Cherokee $x$ Doko

FC $31.665 \times$ Doko

Ivaí $x$ Doko

Mammmoth Yellow $x$ Doko

Majós X Doko

PI 165. $672 \times$ Doko

Tadacha $\times$ Doko

IAC Sta Maria $702 \times$ Doko

Stwart x Doko número de linhagens $F_{7}$ avaliadas \begin{tabular}{l}
1 \\
5 \\
1 \\
2 \\
2 \\
4 \\
1 \\
7 \\
6 \\
3 \\
2 \\
1 \\
2 \\
1 \\
1 \\
1 \\
5 \\
2 \\
4 \\
3 \\
1 \\
3 \\
2 \\
6 \\
1 \\
1 \\
4 \\
1 \\
3 \\
1 \\
5 \\
6 \\
5 \\
2 \\
3 \\
2 \\
2 \\
3 \\
6 \\
4 \\
\hline
\end{tabular}
A freqüência média dos 14 gêneros de fungos nas 116 linhagens de soja obtida, estão dispostos na Tabela 2.

TABELA 2 - Percentagem média de ocorrência de fungos em grãos de soja (Glycine max (L.) Merril) tipo alimento. UEL, Londrina, $\mathrm{Pr}$. 1994.

\begin{tabular}{lr}
\hline FUNGO & $\mathbf{X}$ \\
\hline Aspergillus sp. & $40.37 \%$ \\
Penicillium sp & $18.66 \%$ \\
Fusarium sp & $12.44 \%$ \\
Trichoderma sp & $0.98 \%$ \\
Alternaria sp & $0.24 \%$ \\
Rhizopus sp & $5.71 \%$ \\
Colletotrichum dematium var. truncata & $0.38 \%$ \\
Verticillium sp & $1.52 \%$ \\
Curvularia sp & $0.10 \%$ \\
Nigrospora sp & $0.03 \%$ \\
Phomopsis sojae & $0.08 \%$ \\
Cercospora sp & $1.87 \%$ \\
Diplodia sp & $0.01 \%$ \\
Monilia sp & $0.46 \%$ \\
\hline
\end{tabular}

Os gêneros Aspergillus, Penicillium e Fusarium, todos produtores de toxinas, se destacaram, manifestando freqüência média superior a $12 \%$. O gênero Aspergillus spp. ocorreu em $100 \%$ dos genótipos avaliados, apresentando uma freqüência média de $40,37 \%$, procedido por Penicillium spp. (18,66\%) e Fusarium spp. (12,44\%). A alta percentagem notificada para os três fungos considerados, possivelmente ocorreu devido à incidência, em demasia, de chuvas no momento da colheita. Tais resultados confirmam aos obtidos por LURA et al. (1994), no qual o gênero Aspergillus se destacou com incidência de $60,3 \%$, Penicillium $(16,10 \%)$ e Fusarium $(7,87 \%)$. Em outro grupo, os gêneros Rhizopus, Verticillium e Cercospora ocorreram em uma freqüência média variável de $1 \%$ a $6 \%$. Em níveis inferiores a $1 \%$, foram detectados os fungos Trichoderma sp., Alternaria sp., Colletotrichum dematium var. truncata, Curvularia sp., Nigrospora sp., Monilia sp.; Phomopsis sojae e Diplodia sp.. Tais patógenos, mesmo em baixa freqüência, também podem ser preocupantes, pois, dependendo da sua epidemiologia, mesmo em baixa quantidade de inóculo, alguns são capazes de causar problemas sérios na lavoura. Confirmando os resultados obtidos, HUSSAIN et al. (1989) isolaram de sementes de soja os fungos: Alternaria $s p$, Colletotrichum truncatum, Curvularia sp., Aspergillus spp., Penicillium spp. e Fusarium spp. 
FIGURA 1 - Microorganismos associados a grãos de soja tipo alimento

(\%) de ocorrência

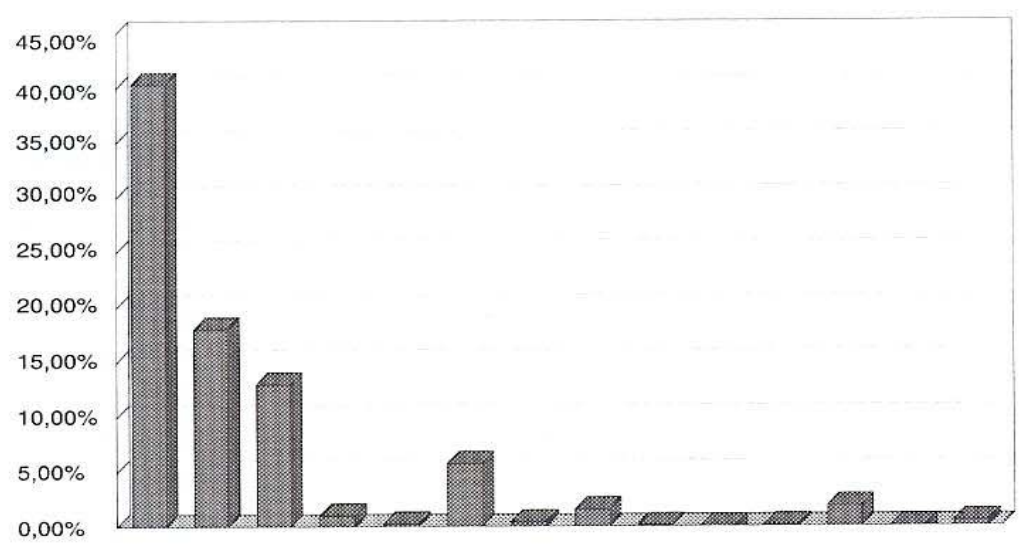

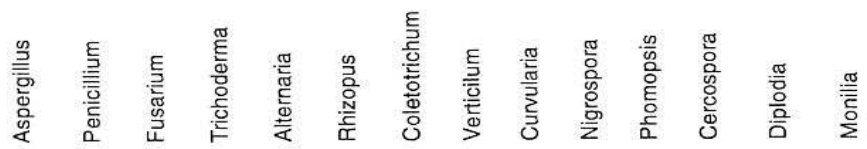

A ocorrência de mais de um patógeno associado aos grãos de soja também foi observada por HOMECHIN et al. (1977).

A questão do uso ou não das sementes e dos genótipos avaliados vai depender de uma série de fatores. Entre eles, a importância econômica do patógeno, ou seja, avaliando não só os danos que promovem em uma dada safra, mas também a sua atuação em cultivos futuros.

A viabilidade do patógeno durante o armazenamento é variável. Os fungos Aspergillus sp., Penicillium sp. e Fusarium sp. em umidade relativa acima dos $60 \%$ aumentam seu potencial de inóculo, promovendo a deterioração do produto além de produção de toxinas que inutiliza o lote contaminado.

Dos 41 cruzamentos realizados, sete se destacaram devido a menor incidência dos fungos Aspergillus spp., Penicillium spp. e Fusarium spp. Tais cruzamentos, presentes na Tabela 3, foram representados por 29 das linhagens avaliadas.

Por outro lado, cinco cruzamentos foram considerados

TABELA 3 - Cruzamentos e as respectivas linhagens $F 7$ que se destacaram com menor incidência de Aspergillus spp. , Penicillium spp. e Fusarium spp.

\begin{tabular}{l|l}
\hline Cruzamentos & Número de linhagens $F_{7}$ \\
\hline
\end{tabular}

(KS \# $3 \times$ Akiyoshi F 7 - 2) $\times$ Doko

$(64-64 \times$ KS 473) $\times$ Doko

PI 165.676 x Doko

Late Giant x Doko

Aliança Preta $\times$ Doko

(TN\# 4 × PI 230.970 F 7 - 4 ) x Doko

Biloxi $252 \mathrm{~N} \times$ Doko inaptos e impróprios, em termos fitopatológicos, para o consumo humano, pois apresentaram alta incidência de algum dos fungos micotoxigênicos mencionados. $O$ cruzamento que apresentou resultados mais negativos foi Wolverine $x$ Doko, com incidência média de $85,2 \%$ de Aspergillus spp., 58,5\% de Penicillium spp. e $26,57 \%$ de Fusarium spp. nas sete linhagens que a representa. Esses cinco cruzamentos, foram representados por 17 linhagens, como mostra a Tabela 4.

\section{CONCLUSŌES}

\begin{tabular}{l} 
TABELA 4 - Cruzamentos e as respectivas linhagens F 7 \\
que se destacaram com maior incidência de \\
Aspergillus spp., Penicillium spp, Fusarium spp., \\
Phomopsis sp. e Alternaria sp. \\
\hline \multicolumn{2}{|l}{ Cruzamentos } & Número de linhagens \\
\hline
\end{tabular}

PI $243.514 \times$ FT - 2

Hogyoku X Doko

Wolverine $x$ Doko

Hampton x Doko (> incidência de Alternaria sp.)

Pluto $x$ Doko ( > incidência de Phomopsis sp.)

1
2
7
4
3

1 - Nas 116 linhagens puras avaliadas observou-se alta variabilidade de incidência dos gêneros Aspergillus spp.; Penicillium sp., Fusarium sp. e outros microrganismos.

2 - Dos 41 cruzamentos efetuados, sete apresentaram incidência relativamente baixa de Aspergillus spp., Penicillium sp. e Fusarium spp., portanto, sendo promissores no processo de melhoramento de soja tipo alimento.

3 - Dos 41 cruzamentos, três manifestaram alta incidência relativa de Aspergillus spp., Penicillium spp. e Fusarium spp., principalmente o cruzamento Wolverine $x$ Doko. Um cruzamento com incidência relativamente alta de Alternaria $s p$. e outro com alta incidência de Phomopsis sp. foram também descartados, já que tais microrganismos começam a se destacar pela produção de toxinas.

4 - Houve favorecimento do desenvolvimento dos fungos produtores de toxinas, nos genótipos de soja estudados e colhidos a campo, em função da ocorrência de chuvas no período da colheita.

BIZZETTO, A.; LEVY, R. M.; HOMECHIN, M. et al. 
Sanitary quality of the soybeans grain food type. Semina: Ci. Agr., Londrina, v.17, n.1, p. 9-14. , mar. 1996.

ABSTRACT: The pourpouse was amount the incidence of fungus in soybean grains proceding for crossing of food soybean type with grain soybean type. The food soybean type comprehend the big seed soybean, whom one hundred wheigth seed (OWS) are like or bigger than 20 grams (vegetable soybean type) and little soybeans seed, whom one hundred weight seed are smaller than 10 grams (soybean for consume as sprouts or natto type). The grain soybean type has a middle size, with (OWS) between 10 and 19 grams, and it is for industry to produce oil and bran. The seed sample of 116 different pure lines were cropped in camp in 1993 and appraised in Blotter Test Method. It was detected the folowing fungus: Aspergillus sp. (40,37\%); Penicillium sp. (18,66\%); Fusarium sp. (12,44\%); Rhizopus sp. (5,71\%); Cercospora sp. (1,87\%) and Verticillium sp. (1,52\%). Bellow of 1,5\% were detected the folowing fungus: Alternaria sp.; Colletotrichum sp.; Trichoderma sp.; Curvularia sp.; Nigrospora sp.; Phomopsis sp.; Diplodia sp. and Monilia sp. In a improvment genetics program for soybean to human consume special atention should be spend to microrganisms like Aspergillus sp.; Penicillium sp. and Fusarium sp.; once that they are capable to produce toxins that may be prejudicial for human beings and animals.

KEY-WORDS: Fungus, Seeds, Soybean, Food.

\section{REFERÊNCIAS BIBLIOGRÁFICAS}

AMARAL, H. M. DO GOTO, R., VICTOR, O. Efeito da flora fungica na qualidade de sementes de soja armazenadas em condições ambientais. Fitopatol. Bras., v.11, n.2, p.133, res. 110, Jun. 1986.

$\mathrm{BOSCH}, \mathrm{U}$., MIROCHA, C. J. Toxin production by Fusarium species from Sugar Beets and natural ocorrence of Zearalenone in Beets and fiber. Appl. Environ. Microbiol., v.58, p.3223, 1992.

BRANCÃO, N., AVOZANI, O. A., DORNELLES, G. L. B. Avaliação de fungos patogênicos associados as sementes de soja (Glycine $\max ($ L.) Merrill). Fitopatol. Bras., v.13, p.145, 1988.

CLEAR, R. M., NOWICKI, T. W., DAUN, J. K.. Soybean seed discoloration by Alternaria spp. and Fusarium spp., effects on quality and production of fusariotoxins. Canadian Journal of Plant Pathology., v.11, n.3, p.308-312, 1989.

CLEUSTROM, G., LJUNGREEN, H., TEGELTROM, S., TIDEMAM, K. Production of aflatoxin by an Aspergillus flavus isolated cultured under a limited oxygen supply. Appl. Environ. Microbiol., v.46, p.400$405,1983$.

DIENER, U. L., COLE, R. J., SANDERS, T. H., PAYNE, G. A., LEE, L. S., KLICH, M. A. Epidemiology of aflatoxin formation by Aspergillus flavus. Annu. Rev. Phytopathol., v.25, p.249-270, 1987.

DOEHL̇ERT, D. C., WICKLOW, D. T., GARDNER, N. W. Evidence implicating the lipoxigenase pathway in proving resistence to soybeans againstAspergillus flavus. Phytopathology, v.83, p.1473$1477,1993$.

DUTTON, M. F., WESTLAKE, K. Ocurrence of mycotoxins in cereals and animals feedstuffs in Natal, South Africa. Journal of the Association of Official Analytical Chemists, v.68, n.5, p.839-842, 1985.

FRISUAD, J. C., FILTENBORG, O. Classification of Terverticillate Penicillia based on profiles of micotoxins and other secondary metabolites. Appl. Environ. Microbiol., v.46, p.1301-1310, 1993.

HENNING, A., YORINORI, J. T., FRANÇA NETO, J. B., KRZYZANOWSKI, F. C. Transmissibilidade e Controle de Principais Patógenos em sementes de soja. (EMBRAPA/CNPSo). Fitopatol. Bras., v.17, n.2, p.171, ago. 1992.

HOMECHIN, M., YORINORI, J. T., MENEZES, J. R. Patógenos de soja transmitidos pela sementes no Estado do Paraná. (EMBRAPA CNPSo). Fitopatol. Bras., n.2, p.79-80, 1977.

HUSSAIN, S., HASSAN, S.; KHAN, B. A.; HUSSAIN, S., HASSAN, $S$. Seed borne mycoflora of soybean in the North West Frontier Province of Pakistan. Sarhad Journal of Agriculture, v.5, n.4, p.421424, 1989.

IMWIDTHAYA, S., ANUKARAHANONTA, T., KOMOLPSIS, P. Bacterial, fungi and aflatoxin contamination of cereal and cereal products in Bangkok. Journal of the Medical Association of Thailand, v.70, n.7, p.390-395, 1987.

LAZZARI, F. A. Umidade, Fungos e Micotoxinas na Qualidade de Sementes, Grãos e Rações. [Curitiba]: Universidade Federal do Paraná, 1995. p.134,

LEMKE, P. A., DAVIS, N. D., CREECH, G. W. Direct visual detection of Aflatoxina synthesis by minicolonies of Aspergillus species. Appl. Environ. Microbiol., v.55, p.1808-1810, 1989.

LURA, M. C. E., GONZALEZ, A. M., BECCARIA, A. J., LATORRE, M. G. ; NEPOTE, A. F., RICO, M. Aislamento, identification y determinacion de la capacidad toxicogenica de hongos contaminantes de cereales y productos alimentos. Catedra de Microbiologia General, Fac. De Bioquimica y Ciencias Biologica, Univ. Nacional del Litoral, Argentina. Boletin Micologo, v.9, n.1/2, p.103-108, 1994.

MARAsAs, W. F. O., NELSON, P. E., TOUSON, T. A. Toxigenic Fusarium species, identity and micotoxicology. Pensylvania State University Press, University Park, 1984.

PITTIS, J. E., MACHADO, J. C. Analysis of micoflora of matter removed during of soybeans seeds. Fitopatol. Bras., v.12, p.408-410, 1987.

SHURTLEFF, M. C. Compendium of corn disease. 20 ed. St Paul : American Phytothological Society, 1980. 105p.

SINCLAIR, J. B., SHURTLEFF, M. C. Compedium of soybean diseases. Minnesota: American Phytopathological Soc. St Paul, 1975. 70p.

ULLSTRUP, A. J. Corn diseases in the United Sates and their control. 
Washington, USDA, 1966. 4p. (Agriculture Society, 105p., 1980).

VELLO, N. A. Ampliação da base genética do germoplasma e melhoramento da soja na ESALQ-USP. In: CÂMARA, G. M. S. ; MARCOS FILHO, J., OLIVEIRA,E. A. M. I Simpósio sobre cultura e produtividade da soja. Piracicaba, SP. FEALQ, 1992. p.60-81.

VIRGINIA, E., PINTO, F., VAAMONDE, G., BRIZZIO, S. B., APRO, $\mathrm{N}$. Aflatoxin production soybeans varieties grown in Argentina. Journal of Foof Protection., v. 54, n 7, p.542-545, 1991.

YORINORI, J. T., GARCIA, A. Danos causados por Cercospora sojina Hara nas sementes do cultivar de soja. Bragg. Londrina, PR: CNPSO/EMBRAPA, 1971.

YORINORI, J. T. Diferenciação entre Phomopsis da queima da haste e da vagem e do cancro da haste em sementes de soja através do "Blotter test". Fitopatol. Bras., v.16, n.2, jun. 1991.

YORINORI, J. T. Cancro-da-haste (Diaporthe phaseolorum f. sp. meridionalis) uma série ameaça a cultura de soja no Brasil. Fitopatol. Bras., v.15, n.2, p.124, 1990.

WETZEL, M. M. V. da S. Fungos de Armazenamento. In: SOAVE, J., WETZEL, M. M. V. da S. Patologia de sementes. Campinas, SP: Fundação Cargill, 1987. p.260-270.

WICKLOW, D. T., HESSELTINE, C. W., SHOTWELL, O. L., ADAMS, G. L. Interference competition and aflatoxin levels in corn. Phytopathology, v.70, p.761, 1980. 